\title{
The Excitation and Rapid Damping of Coronal Loop Oscillations Observed from the TRACE
}

\author{
A.M. Uralov \\ Institute of Solar-Terrestrial Physics, Irkutsk, Russia, email: uralov@iszf.irk.ru
}

TRACE (Transition Region and Coronal Explorer) observations of transverse oscillations in coronal loops have prompted a number of questions to which there are no convincing answers as yet. Only the conclusion that the appearance of oscillations reflects the response of the magnetosphere of an active region to an internal or external magnetohydrodynamic (MHD) disturbance seems beyond question. The sources of such disturbances are solar flares or filament eruptions. The putative correspondence of the observed oscillations to the axisymmetric fundamental kink mode of a single coronal loop raises two questions at once: how to excite this mode and how to explain the rapid damping of the oscillation.

We suggest a way of self-consistently solving these questions. The excitation of oscillations and their rapid damping can be naturally explained by the appearance of a dispersion wake behind the moving MHD pulse. The phenomenon is completely described in terms of ideal magnetohydrodynamics. Oscillations are excited on the dispersion branch of fast magnetoacoustic waves, which propagate mainly across the magnetic field lines whose ends are rigidly fixed. The rapid damping of the observed oscillations is governed by the dispersion spreading of the pulse of these waves. The oscillation period is close to the period of the fundamental mode. Dissipative processes (including resonant absorption) attributable to the nonideality of the plasma and the coronal-loop footpoints play no fundamental role.

The energy of the wave wake increases with distance from the MHD pulse initiation site and can be comparable to the wave energy of the initial disturbance. The coronal magnetic flux of all or part of the magnetosphere of an active region is involved in the oscillatory motion. The presence of isolated, denser coronal EIT loops modifies the solution but does not change its global character. "Heavy" coronal loops must be forcedly displaced by the environment oscillatory motion if inequality is satisfied: $\left(\frac{d}{L}\right)^{2}\left(\frac{\rho_{i n}}{\rho_{e x}}-1\right)^{2}<<1$, where $\rho_{i n}$ and $\rho_{e x}$ - are the plasma densities inside and outside of the loop whose length and diameter are $L$ and $d$. This inequality is satisfied with a margin, and, therefore, oscillations observed from TRACE are, to a greater extent, determined not only by the intrinsic parameters of the oscillating loop itself but by the parameters of the transient wave process in the surrounding magnetospheric area of an active region. (Astronomy Letters, vol.29, No.7, 2003, p.486. Translated from Pis'ma v Astronomicheski'i Zhurnal, vol.29, No.7, 2003, p.552)

\section{Acknowledgements}

This research is supported by the Russian Ministry of Science grant 477.2003.2. 


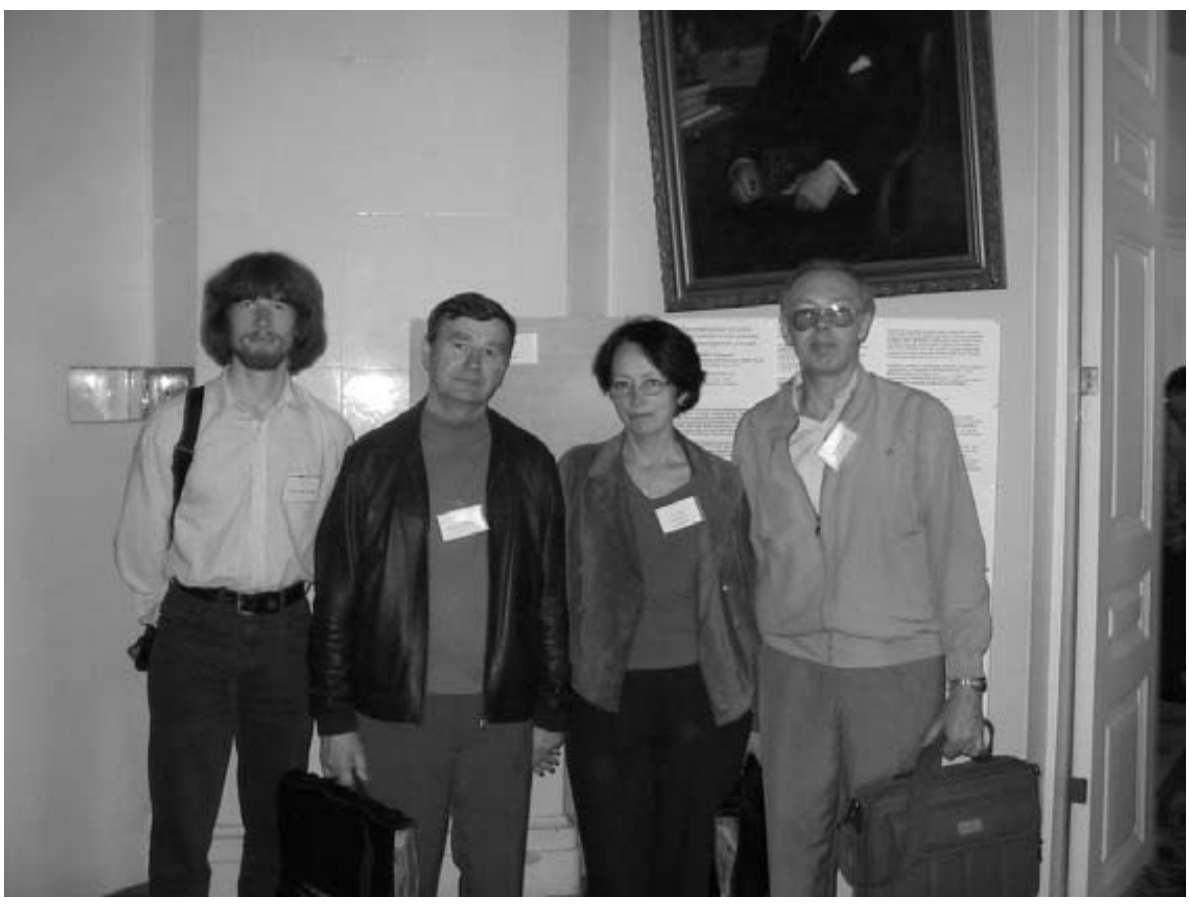

Olexa Andrienko (Ukraine), Roman Kostyk (Ukraine), Natalia Shchukina (Ukraine) and Eugene Miletsky (Russia)

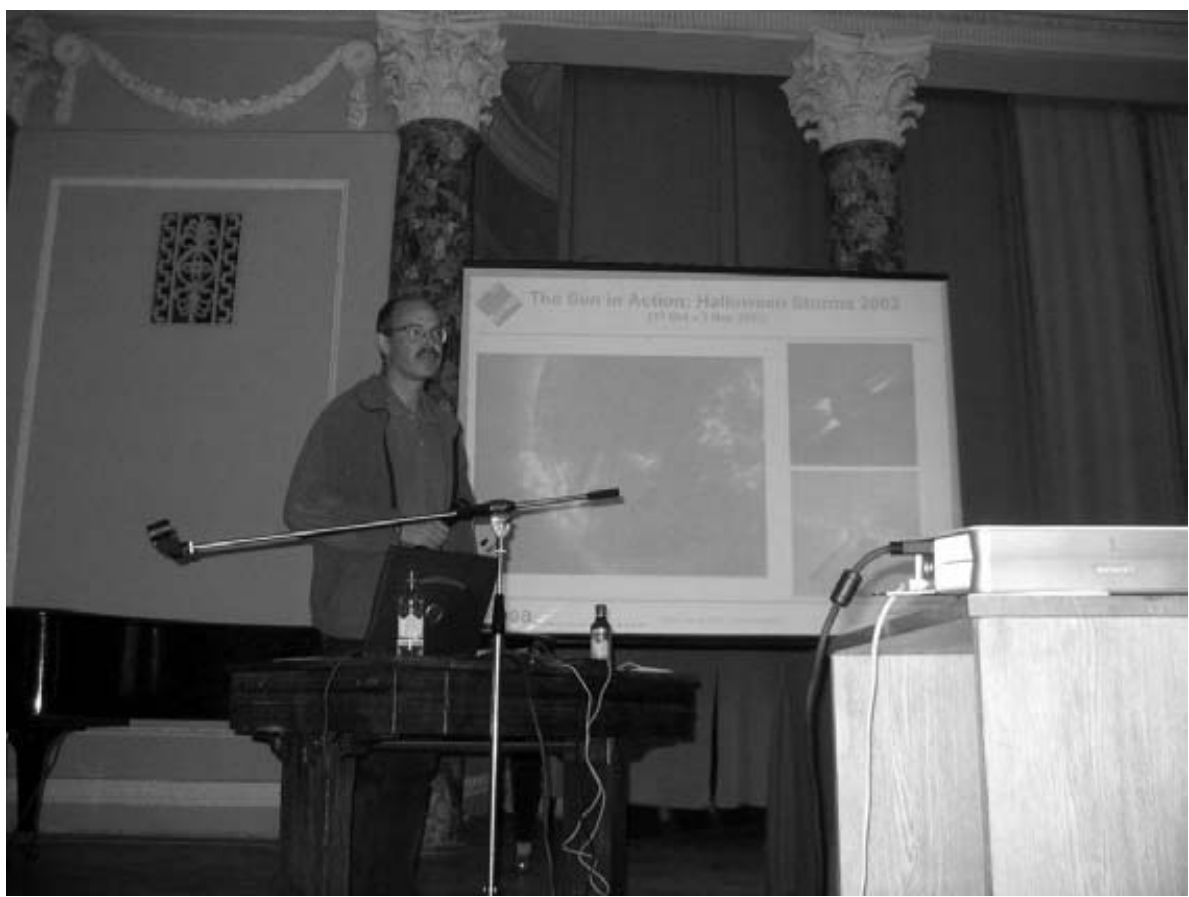

Bernhard Fleck (ESA) 